\title{
Labyrinthe
}

20 | 2005 (1)

La Cognition

\section{Littérature et sciences cognitives}

\section{Sylvain Prudhomme}

\section{(2) OpenEdition}

Journals

Édition électronique

URL : http://journals.openedition.org/labyrinthe/761

DOI : 10.4000/labyrinthe.761

ISSN : 1950-6031

Éditeur

Hermann

Édition imprimée

Date de publication : 20 avril 2005

Pagination : 93-97

Référence électronique

Sylvain Prudhomme, "Littérature et sciences cognitives », Labyrinthe [En ligne], 20 | 2005 (1), mis en ligne le 08 juillet 2008, consulté le 20 avril 2019. URL : http://journals.openedition.org/labyrinthe/761 DOI : 10.4000/labyrinthe.761

Propriété intellectuelle 


\title{
LITTÉRATURE ET SCIENCES COGNITIVES
}

\author{
Sylvain PRUdHOMme \\ prudhommesy@wanadoo.fr
}

Le développement des sciences cognitives offre aujourd'hui aux études littéraires, traditionnellement rétives aux sciences, l'occasion précieuse de repenser leurs relations avec la démarche et les méthodes scientifiques. Le cognitivisme pourrait, en théorie, leur venir en aide à deux niveaux: appréhension de l'acte d'écriture d'abord, de l'opération de lecture ensuite - chacun de ces processus mobilisant des compétences spécifiques et requérant une chaîne d'opérations mentales que la psychologie cognitive, notamment, devrait pouvoir s'attacher à décrire. Or, les explorations conduites dans l'une et l'autre de ces voies montrent des limites qui mettent en doute la fécondité d'une telle utilisation.

Le récent ouvrage Cognition et création, publié sous la direction de Mario Borillo et Jean-Marie Goulette, offre un bon exemple de tentative d'exploration cognitiviste du psychisme créateur, visant à «pénétrer au plus intime du processus de création de l'œuvre d'art, à le décrire, à l'expliquer dans les termes d'une transdisciplinarité qui associe, autour du concept de computation, des disciplines aussi diverses que les neurosciences, la psychologie, les sciences du langage, enfin l'informatique et les mathématiques ${ }^{1}{ }^{\prime}$. Les analyses s'y succèdent, tantôt effectivement consacrées à la description des étapes successives du processus de conception - modélisé sous la forme d'une série de corrections progressivement apportées, par va-et-vient entre croquis et images mentales, à un modèle initial -, tantôt dérivant vers des questions importantes mais connexes comme celle de l'enseignabilité de la création, celle de l'assistance informatique à la conception, quand ce n'est pas vers la stigmatisation stérile des résistances rencontrées par

1. Mario Borillo et Jean-Marie Goulette (dir.), Cognition et création, p. 12. 
le point de vue cognitiviste au sein du monde artistique et critique ${ }^{2}$. Fait symptomatique, à aucun moment la littérature n'est évoquée. Le champ étudié se trouve de facto réduit aux arts plastiques et surtout à l'architecture, en accord avec le privilège tacitement accordé par la psychologie cognitive au visuel. Cet oubli, volontaire ou non, reflète la difficulté d'aborder le cas littéraire avec les mêmes outils que celui des Beaux-Arts : la spécificité du matériau verbal, son épaisseur signifiante rendent en effet caducs les modèles couramment employés pour décrire le processus créateur, qui le réduisent en général à un problème de reconnaissance et de production de formes (en faisant une impasse tout aussi problématique, mais moins visible peut-être que lorsqu'il s'agit du langage, sur l'épaisseur du matériau pictural, sculptural ou architectural).

Quelques exemples existent certes d'études qui s'efforcent de partir avant tout des textes, et notamment du lexique. Telle est l'ambition de l'une des plus significatives sans doute, celle consacrée à Shakespeare par M. T. Crane: «Découvrir dans chaque pièce un réseau de mots relié à des métaphores spatiales et reflétant dans ses grandes lignes certains des schémas et des points clés du lexique mental de Shakespeare ${ }^{3}$.» On retrouve un vrai ancrage dans le texte, qui permet la mise au jour d'enjeux essentiels à la compréhension des pièces étudiées. Mais l'approche ici n'est plus cognitive qu'au sens littéral, en tant qu'elle envisage le texte comme la manifestation d'un psychisme singulier dont il est possible de reconstruire, par induction, certains traits caractéristiques idéaux. Plus la moindre influence des simulations informatiques ni même du vocabulaire et des outils conceptuels chers à la psychologie cognitive: on est face à une critique d'inspiration avant tout littéraire, qu'il faudrait plutôt rapprocher, si l'on devait à tout prix la situer, de celle pratiquée par le Jean-Pierre Richard des Onze Études sur la poésie moderne, notamment dans les pages qu'il consacre à la vision pongienne du monde et à la récurrence du schème descriptif de l'« à la fois » dans Le Parti pris des choses ${ }^{4}$.

\footnotetext{
2. Roger Pouivet, « Conception cognitive de l'art et création artistique », dans Cognition et création, op. cit., p. 277-287.

3. Mary Thomas Crane, Shakespeare's Brain. Reading with Cognitive Theory, p. 4 (c'est nous qui traduisons). Voir aussi Meera Tamaya, An Interpretation of Hamlet Based on Recent Developments in Cognitive Studies.

4. Jean-Pierre Richard, Onze Études sur la poésie moderne, p. 217 et suivantes.
} 
On en vient donc à ce paradoxe que les tentatives les plus convaincantes d'exploration «cognitive» du texte littéraire sont celles qui, en réalité, se passent quasiment de tout recours direct à la psychologie cognitive. Indice d'une insuffisance du paradigme cognitiviste face à l'objet littéraire? C'est ce que semblent confirmer les études consacrées à la lecture, elles aussi en butte à de nombreuses difficultés. Qu'elles relèvent de la psychologie cognitive et s'attachent à décrire le détail des opérations mentales du lecteur, ou qu'elles soient d'ordre sémantique et se focalisent sur le texte et la manière dont le sens s'y construit, elles se maintiennent en effet à un niveau de généralité qui les empêche de prendre pour objet, sinon la fameuse et controversée littérarité en tant que telle, du moins la différence spécifique de tel ou tel texte littéraire singulier par rapport à un autre - sa façon particulière de signifier, les effets de lecture singuliers qu'il produit - et à plus forte raison par rapport à un texte non littéraire. Longtemps exclusivement absorbé par la recherche d'universaux, le cognitivisme commence tout juste à quitter le plan du langage pour descendre vers la diversité des langues: c'est dire si l'étude de la diversité des textes, plus inépuisable encore, est pour le moment loin de ses préoccupations.

Il ne faut donc pas seulement chercher les raisons de la nonrencontre entre sciences cognitives et littérature dans la réticence, souvent incriminée, des belles-lettres à la technicité, ni même dans un effroi devant la prétendue «inversion du concept théologique de création $^{5} \gg$ que supposerait la perspective cognitiviste. L'absence d'articulation véritablement convaincante entre les deux domaines tient aussi aux limites inhérentes au cognitivisme actuel: non seulement au degré de généralité très élevé des opérations qu'il se donne pour objet, mais aussi à une autre insuffisance, peut-être plus profonde et plus inquiétante quant à la possibilité d'une entente avec la littérature: sa conception même de la connaissance et du sens, que François Rastier qualifie de «théorie du reflet»: «La connaissance est [pour le cognitivisme] un processus de représentation conçu comme une traduction symbolique: le monde est composé de choses et d'états de choses; les connaissances sont des représentations symboliques de ces choses et de ces états de choses $^{6}$.» En découle une conception exclusivement dénotative du

\footnotetext{
5. Roger Pouivet, art. cit., p. 277.

6. François Rastier, Sémantique et sciences cognitives, p. 38.
} 
sens: au symbole correspond la chose ou l'état de chose, et réciproquement, dans une univocité qui interdit tout jeu. Nul besoin d'insister sur l'incompatibilité foncière d'une telle approche de la signification avec la démarche littéraire, qui n'a cessé de revendiquer connotation et polysémie comme traits caractéristiques du texte littéraire, sinon comme pierre de touche de toute littérarité.

Si bien que, en définitive, c'est peut-être au sein non pas des études littéraires mais des pratiques d'écriture elles-mêmes qu'il faut rechercher les traces les plus probantes d'une influence de l'analogie esprit/ordinateur. Parmi les nombreux et multiformes «écrits d'écran» apparus depuis une décennie se sont en effet développés des programmes qui, à la différence de la majeure partie de la littérature électronique pour laquelle l'informatique n'est qu'un moyen d'enrichir et de renouveler les formes d'expression, ont véritablement cherché à substituer la machine à l'auteur: les Générateurs automatiques de texte. En visitant le site www.charabia.net, créé par Francisco Reyes, on découvre différents moteurs capables de générer à volonté haïkus de la plus pure fabrique, «injures à la manière du capitaine Haddock», et «charabia philosophique».

On aurait pourtant tort d'assimiler ces prouesses de programmateurs aux expériences de simulation artificielle conduites par les cognitivistes. Loin de chercher à reproduire les différentes étapes du psychisme créateur, les générateurs de texte se concentrent, grâce à une méthode combinatoire que Francisco Reyes dévoile dans une rubrique de son site $^{7}$, sur l'imitation du résultat du processus de production. Autrement dit l'analogie esprit/ordinateur se réduit à sa forme minimale : elle ne porte que sur les sorties obtenues. L'ambition n'est nullement scientifique: il ne s'agit pas de connaître ni de comprendre, mais bien plutôt de démystifier, de railler en montrant que la machine fait aussi bien, sinon mieux - car la qualité des haïkus est surprenante que l'auteur. Geste anodin pour ce qui nous regarde ici? Nullement!

\footnotetext{
7. L'ordinateur dispose en réalité, pour chaque syntagme du haïku ou de l'injure à générer (adjectif, substantif, complément du nom, etc.), d'une liste d'éléments interchangeables entre lesquels, sur commande, il choisit de façon aléatoire, de sorte que chaque nouvel énoncé est en fait construit par décomposition/recomposition du précédent, grâce à une série de substitutions partielles simultanées. Méthode assez simple, mais merveilleusement habile dans son utilisation de la double dimension, syntagmatique et paradigmatique, de la langue.
} 
Preuve supplémentaire, s'il était besoin, que ce n'est pas (ou pas seulement) par attachement au mythe du génie créateur que la littérature et les études littéraires continuent de se dérober aux sciences cognitives.

\section{ÉLÉMENTS DE BIBLIOGRAPHIE}

Borillo Mario, Goulette Jean-Marie (dir.), Cognition et création. Explorations cognitives des processus de création, Bruxelles, Mardaga, 2002.

CRANE Mary Thomas, Shakespeare's Brain. Reading with Cognitive Theory, Princeton and Oxford, Princeton University Press, 2001.

Meyer Michel, Langage et littérature, Paris, Puf, coll. "Quadrige», 2001.

RASTIER François, Sémantique et sciences cognitives, Paris, Puf, coll. «Formes sémiotiques», 1991.

-, Arts et sciences du texte, Paris, Puf, coll. «Formes sémiotiques», 2001.

ReYes Francisco, www.charabia.net

RichARD Jean-Pierre, Onze Études sur la poésie moderne, Paris, Seuil, coll. «Points Essais », 1964.

TAMAYA Meera, An Interpretation of Hamlet Based on Recent Developments in Cognitive Studies, Lewiston, The Edwin Mellen Press, 2001. WiJsen Louk M. P. T., Cognition and Image Formation in Litterature, Frankfurt am Main, P. D. Lang, 1980. 